Mathematical Modelling and Analysis

Volume 17 Number 2, April 2012, 190-202

http://dx.doi.org/10.3846/13926292.2012.660889

(C) Vilnius Gediminas Technical University, 2012
Publisher: Taylor\&Francis and VGTU

http://www.tandfonline.com/TMMA

Print ISSN: 1392-6292

Online ISSN: 1648-3510

\title{
Approximate Solution of Nonlinear Multi-Point Boundary Value Problem on the Half-Line*
}

\author{
Jing Niu ${ }^{a, c}$, Ying Zhen $\operatorname{Lin}^{b}$ and Chi Ping Zhang ${ }^{a}$ \\ ${ }^{a}$ Harbin Institute of Technology \\ 150001 Harbin, HeiLongJiang, China \\ ${ }^{b}$ Harbin Institute of Technology at Weihai \\ 264209 ShanDong, China \\ ${ }^{c}$ Harbin Normal University \\ 150025 Harbin, HeiLongJiang, China \\ E-mail(corresp.): niujing1982@gmail.com \\ E-mail: liliy55@163.com; zcp@hit.edu.cn
}

Received April 8, 2011; revised December 28, 2011; published online April 1, 2012

\begin{abstract}
In this work, we construct a novel weighted reproducing kernel space and give the expression of reproducing kernel function skillfully. Based on the orthogonal basis established in the reproducing kernel space, an efficient algorithm is provided to solve the nonlinear multi-point boundary value problem on the half-line. Uniformly convergence of the approximate solution and convergence estimation of our algorithm are studied. Numerical results show our method has high accuracy and efficiency.

Keywords: multi-point boundary value conditions, half-line, approximate solution, weighted reproducing kernel space.
\end{abstract}

AMS Subject Classification: 34K28; 30E25; 34B10.

\section{Introduction}

We are concerned with the approximate solution of the following multi-point boundary value problem posed on the positive half-line [13]:

$$
\begin{cases}x^{\prime \prime}(t)-p x^{\prime}(t)-q x(t)=f(t, x(t)), & t \in[0, \infty), \\ \alpha x(0)-\beta x^{\prime}(0)-\sum_{\mathrm{i}=1}^{n} k_{i} x\left(\xi_{i}\right)=a_{0}, & \lim _{\mathrm{t} \rightarrow+\infty} \frac{x(t)}{e^{r t}}=b_{0} .\end{cases}
$$

Here $p, q, \xi_{i}, a, b$ are some nonnegative real numbers, $r \in\left(0, \frac{p+\sqrt{p^{2}+4 q}}{2}\right], f$ is a continuous function and $\alpha, \beta, k_{i} \geq 0$ satisfy $\alpha^{2}+\beta^{2} \neq 0$.

* The first author's work was supported by Youth Foundation of Heilongjiang Province (QC2010036) and also supported by Fundamental Research Funds for the Central Universities ( No.HIT.NSRIF.2009050), Academic Foundation for Youth of Harbin Normal University(11KXQ-04) and also by 10KXQ-05 
Boundary value problems(BVPs) on the half-line arise in various applications of physical phenomena and in chemistry, epidemiology $[1,10,11]$. Thus, there have been so much work devoted to the existence of solutions for boundary value problem on the half-line, see, for example, [2, 7, 9] and the references therein. The main methods used to tackle these problems are upper and lower solutions techniques $[9,12,14]$, fixed point theorems in special Banach spaces and recent fixed point theory on cones of Banach spaces $[5,6,10,13]$. They all discussed the existence and multiplicity of positive solutions of nonlinear differential equations with BVPs on the half-line. The aim of the paper is to seek a numerical solution of such BVPs in a reproducing kernel Hilbert space.

Recently, Cui and Lin [4] presented an iterative reproducing kernel method (RKM) used for obtaining approximate solutions of differential and integral equations with initial or boundary value conditions. For example, a class of linear Volterra integral equation with weakly singular kernel is discussed in [3]. Lin and Zhou [8] have solved the nonlinear pseudoparabolic equations with nonlocal boundary conditions numerically. Two point boundary value problems of $2 m$ th-order nonlinear differential equations are considered in [15]. However, due to the multi-point boundary value conditions with a limit form in Eq. (1.1), especially on the half-line, it is difficult to find the corresponding reproducing kernel space by applying traditional RKM. The aim of our work is to extend RKM to the nonlinear multi-point boundary value problem on the half-line. One important improvement is that the range space of our method is $L^{2}[0,+\infty)$ on the half-line, while traditional RKM is $L^{2}[a, b]$ of finite interval $[3,8,15]$. Actually, it is an essential difficulty for discussing the problem with this transition. Meanwhile, because traditional RKM [3, 4, 8, 15] are all based on reproducing kernel space included bounded functions. The emphasis of the result is that we construct a reproducing kernel space included unbounded functions so as to overcome difficulties with $\lim _{t \rightarrow+\infty} \frac{x(t)}{e^{r t}}=b_{0}$. Therefore, the application of our method is more extensive than traditional RKM.

Motivated by all the works above, firstly the authors successfully construct a novel weighted reproducing kernel space which includes boundary conditions with the limit form and give the expression of the reproducing kernel function. Secondly, we establish an orthogonal basis in the weighted reproducing kernel space and estimate the complexity of the orthogonal basis. And then the approximate solution is obtained by the iteration technique. Uniformly convergence and the error estimation are provided for the numerical scheme. Finally, the numerical results are given to demonstrate the effectiveness and accuracy of the method. Furthermore, the numerical algorithms introduced in this study can be well suited for handling nonlinear differential equations with various multi-point boundary conditions on the half-line.

\section{A Novel Weighted Reproducing Kernel Space}

Definition 1. [4] Let $H$ be a Hilbert function space on a set $X$. $H$ is called a reproducing kernel space if and only if for any $x \in X$, there exists a unique function $R_{x}(y) \in H$, such that $\left\langle f, R_{x}\right\rangle=f(x)$ for any $f \in H$. Meanwhile, 
$R(x, y) \triangleq R_{x}(y)$ is called a reproducing kernel function.

Due to the complex boundary conditions of (1.1), the following weighted reproducing kernel space is constructed firstly.

Definition 2. $H \triangleq H\left([0,+\infty), e^{-r t}\right)=\left\{x(t) \mid x^{\prime \prime}(t)\right.$ is absolutely continuous and $\left.\lim _{\mathrm{t} \rightarrow \infty} \frac{x(t)}{e^{r t}}=0, \int_{0}^{+\infty} e^{-r t}\left[x^{(3)}(t)\right]^{2} d t<+\infty\right\}$. The inner product is defined respectively by

$$
\langle x(t), y(t)\rangle_{H}=\sum_{i=0}^{2} x^{(i)}(0) y^{(i)}(0)+\int_{0}^{+\infty} e^{-r t} x^{(3)}(t) y^{(3)}(t) d t .
$$

Theorem 1. $H$ is a Hilbert reproducing kernel space and its reproducing kernel function is as following:

$$
R(t, s)= \begin{cases}r_{1}(t, s)=e^{r t} \sum_{i=1}^{3} a_{1 i}(s) t^{i-1}+\sum_{i=4}^{6} a_{1 i}(s) t^{i-4}, & t \leq s, \\ r_{2}(t, s)=e^{r t} \sum_{i=1}^{3} a_{2 i}(s) t^{i-1}+\sum_{i=4}^{6} a_{2 i}(s) t^{i-4}, & s \leq t .\end{cases}
$$

According to the definition of reproducing kernel space, $H$ is a generalization of $[4$, Theorems 1.3.1, 1.3.2] with essentially the same proofs. Therefore, we omit the proof of Theorem 1 .

Theorem 2. The subspace $H_{0} \triangleq H_{0}\left([0,+\infty), e^{-r t}\right)=\{x(t) \mid x(t) \in H$, $\left.\alpha x(0)-\beta x^{\prime}(0)-\sum_{\mathrm{i}=1}^{n} k_{i} x\left(\xi_{i}\right)=0\right\}$ of $H$ is a reproducing kernel space and its reproducing kernel function is

$$
K(t, s)=R(t, s)-\frac{f(t) f(s)}{\alpha f(0)-\beta f^{\prime}(0)-\sum_{i=1}^{n} k_{i} f\left(\xi_{i}\right)}
$$

where $f(t)=\alpha R(t, 0)-\beta \partial_{s} R(t, 0)-\sum_{i=1}^{n} k_{i} R\left(t, \xi_{i}\right)$ and $R(t, s)$ is the reproducing kernel function of $H$.

Proof. Since $H_{0}$ is a closed subspace of reproducing kernel space $H, H_{0}$ is a reproducing kernel space. Next we shall prove that $K(t, s)$ is the reproducing kernel of $H_{0}$. At first we prove that $\alpha f(0)-\beta f^{\prime}(0)-\sum_{i=1}^{n} k_{i} f\left(\xi_{i}\right) \neq 0$. The proof is done by using the contradiction method. In the view of the reproducing property, it is clear that

$$
\begin{aligned}
& \langle f(t), f(t)\rangle_{H}=\left\langle f(t), \alpha R(t, 0)-\beta \partial_{s} R(t, 0)-\sum_{i=1}^{n} k_{i} R\left(t, \xi_{i}\right)\right\rangle_{H} \\
& =\alpha\langle f(t), R(t, 0)\rangle_{H}-\beta\left\langle f(t), \partial_{s} R(t, 0)\right\rangle_{H}-\sum_{i=1}^{n} k_{i}\left\langle f(t), R\left(t, \xi_{i}\right)\right\rangle_{H} \\
& =\alpha f(0)-\beta f^{\prime}(0)-\sum_{i=1}^{n} k_{i} f\left(\xi_{i}\right)=0 .
\end{aligned}
$$

Consequently it follows that $f(t)=0$. Then for any $y(t) \in H$, we get $\alpha y(0)-\beta y^{\prime}(0)-\sum_{i=1}^{n} k_{i} y\left(\xi_{i}\right)=\left\langle y(t), \alpha R(t, 0)-\beta \partial_{s} R(t, 0)-\sum_{i=1}^{n} k_{i} R\left(t, \xi_{i}\right)\right\rangle_{H} \equiv 0$ 
which indicates $y(t) \in H_{0}$. This contradicts that $H_{0}$ is a proper subspace of $H$.

Second, we need to show for any $s \in[0,+\infty), K(t, s) \in H_{0}$. In fact, it follows that

$$
\begin{aligned}
& \alpha K(0, s)-\beta \partial_{t} K(0, s)-\sum_{i=1}^{n} k_{i} K\left(\xi_{i}, s\right)=\alpha R(0, s)-\beta \partial_{t} R(0, s) \\
& \quad-\sum_{i=1}^{n} k_{i} R\left(\xi_{i}, s\right)-\frac{\left(\alpha f(0)-\beta f^{\prime}(0)-\sum_{i=1}^{n} k_{i} f\left(\xi_{i}\right)\right) f(s)}{\alpha f(0)-\beta f^{\prime}(0)-\sum_{i=1}^{n} k_{i} f\left(\xi_{i}\right)} \\
& =\alpha R(0, s)-\beta \partial_{t} R(0, s)-\sum_{i=1}^{n} k_{i} R\left(\xi_{i}, s\right)-f(s)=f(s)-f(s)=0 .
\end{aligned}
$$

Finally, for any $x(t) \in H_{0}$, there holds that

$$
\begin{aligned}
\langle x(t), K(t, s)\rangle_{H}=\left\langle x(t), R(t, s)-\frac{f(t) f(s)}{\alpha f(0)-\beta f^{\prime}(0)-\sum_{i=1}^{n} k_{i} f\left(\xi_{i}\right)}\right\rangle_{H} \\
=x(s)-\frac{f(s)}{\alpha f(0)-\beta f^{\prime}(0)-\sum_{i=1}^{n} k_{i} f\left(\xi_{i}\right)} \\
\quad \times\left\langle x(t), \alpha R(t, 0)-\beta \partial_{s} R(t, 0)-\sum_{i=1}^{n} k_{i} R\left(t, \xi_{i}\right)\right\rangle_{H} \\
=x(s)-\frac{f(s)}{\alpha f(0)-\beta f^{\prime}(0)-\sum_{i=1}^{n} k_{i} f\left(\xi_{i}\right)}\left(\alpha x(0)-\beta x^{\prime}(0)-\sum_{i=1}^{n} k_{i} x\left(\xi_{i}\right)\right) \\
=x(s) .
\end{aligned}
$$

That is, $K(t, s)$ is the reproducing kernel function of $H_{0}$.

\section{The Numerical Method}

According to [13], we know the existence of solutions for the problem (1.1) only if it satisfies the following condition:

(H1) $f$ is continuous such that $f(t, x) \leq c(t)+d(t) x$ for $(t, x) \in[0,+\infty) \times$ $C([0,+\infty),[0,+\infty))$, and $c, d:[0,+\infty) \rightarrow[0,+\infty)$ are continuous functions;

(H2) $\alpha-\beta \lambda_{1}-\sum_{\mathrm{i}=1}^{n} k_{i} e^{\lambda_{1} \xi_{i}}>0$ and $\frac{\left(\alpha-\beta \lambda_{2}\right) \int_{0}^{\infty} d(s) e^{-\lambda_{1} s} d s}{\left(\lambda_{1}-\lambda_{2}\right)\left(\alpha-\beta \lambda_{2}-\sum_{\mathrm{i}=1}^{n} k_{i} e^{\lambda_{2} \xi_{i}}\right)}<1$, where $\lambda_{1}=\frac{p+\sqrt{p^{2}+4 q}}{2}, \lambda_{2}=\frac{p-\sqrt{p^{2}+4 q}}{2}$.

Here the approximate solution of Eq. (1.1) in $H_{0}$ is obtained step by step. At first we homogenize the boundary value conditions of Eq. (1.1) so that it can be discussed in the reproducing kernel space. Next we give an operator equation which is equivalent to Eq. (1.1) and the operator is proved to be bounded. Then a normal orthogonal basis is established. Finally, these are used to successively find the numerical solution in $H_{0}$. 
We observe from boundary conditions that Eq. (1.1) can be transformed to the following form:

$$
\begin{cases}y^{\prime \prime}(t)-p y^{\prime}(t)-q y(t)=g(t, y(t)), & t \in[0,+\infty) \\ \alpha y(0)-\beta y^{\prime}(0)-\sum_{i=1}^{n} k_{i} y\left(\xi_{i}\right)=0, & \lim _{\mathrm{t} \rightarrow+\infty} \frac{y(t)}{e^{r t}}=0 .\end{cases}
$$

Definition 3. $L^{2} \triangleq L^{2}\left([0,+\infty), e^{-\mu t}\right)=\left\{y(t) \mid \int_{0}^{+\infty} e^{-\mu t} y^{2}(t) d t<+\infty\right\}$. The inner product is defined respectively by

$$
\langle y(t), z(t)\rangle_{L^{2}}=\int_{0}^{+\infty} e^{-\mu t} y(t) z(t) d t .
$$

It is easy to see that this $L^{2}$ space is a Hilbert space.

Theorem 3. Let $\mathbb{L}: H_{0} \rightarrow L^{2}$, (LLy) $(t) \triangleq y^{\prime \prime}(t)-p y^{\prime}(t)-q y(t)$. (1) If $\mu>r$, then $\mathbb{L}$ is a bounded linear operator; (2) If (H1), (H2) hold, then $\mathbb{L}$ is invertible.

\section{Proof. (1) Since}

$$
\begin{aligned}
\left|y^{\prime \prime}(t)\right| & \leq\left|y^{\prime \prime}(0)\right|+\int_{0}^{t}\left|y^{(3)}(s)\right| d s \leq\left|y^{\prime \prime}(0)\right|+\sqrt{t}\left(\int_{0}^{t}\left[y^{(3)}(s)\right]^{2} d s\right)^{1 / 2} \\
& \leq\|y\|_{H}+\sqrt{t e^{r t}} \int_{0}^{t} e^{-r s}\left[y^{(3)}(s)\right]^{2} d s \\
& \leq\left(1+\sqrt{t e^{r t}}\right)\|y\|_{H}=n_{t}\|y\|_{H}, \quad n_{t}=1+\sqrt{t e^{r t}} \\
\left|y^{\prime}(t)\right| & \leq\left|y^{\prime}(0)\right|+\int_{0}^{t}\left|y^{\prime \prime}(s)\right| d s \leq\left|y^{\prime}(0)\right|+\int_{0}^{t} n_{s}\|y\|_{H} d s \\
& \leq\|y\|_{H}+\int_{0}^{t} n_{s} d s\|y\|_{H} \leq m_{t}\|y\|_{H}, \quad m_{t}=1+\int_{0}^{t} n_{s} d s \\
|y(t)| & \leq|y(0)|+\int_{0}^{t}\left|y^{\prime}(s)\right| d s \leq|y(0)|+\int_{0}^{t} m_{s}\|y\|_{H} d s \\
& \leq\|y\|_{H}+\int_{0}^{t} m_{s} d s\|y\|_{H} \leq v_{t}\|y\|_{H}, \quad v_{t}=1+\int_{0}^{t} m_{s} d s
\end{aligned}
$$

we readily have

$$
\begin{aligned}
& \|\mathbb{L} y\|_{L^{2}} \leq\left\|y^{\prime \prime}\right\|_{L^{2}}+p\left\|y^{\prime}\right\|_{L^{2}}+q\|y\|_{L^{2}} \\
& \quad \leq \sqrt{\int_{0}^{+\infty} e^{-\mu t} y^{\prime \prime}(t)^{2} d t}+p \sqrt{\int_{0}^{+\infty} e^{-\mu t} y^{\prime}(t)^{2} d t}+q \sqrt{\int_{0}^{+\infty} e^{-\mu t} y(t)^{2} d t} \\
& \leq \sqrt{\int_{0}^{+\infty} e^{-\mu t} n_{t}^{2}\|y\|_{H}^{2} d t}+p \sqrt{\int_{0}^{+\infty} e^{-\mu t} m_{t}^{2}\|y\|_{H}^{2} d t}+q \sqrt{\int_{0}^{+\infty} e^{-\mu t} v_{t}^{2}\|y\|_{H}^{2} d t}
\end{aligned}
$$




$$
\leq\|y\|_{H}\left(\sqrt{\int_{0}^{+\infty} e^{-\mu t} n_{t}^{2} d t}+p \sqrt{\int_{0}^{+\infty} e^{-\mu t} m_{t}^{2} d t}+q \sqrt{\int_{0}^{+\infty} e^{-\mu t} v_{t}^{2} d t}\right)
$$

Notice that, as $\mu>r$, we have

$$
\int_{0}^{+\infty} e^{-\mu t} n_{t}^{2} d t<+\infty, \quad \int_{0}^{+\infty} e^{-\mu t} m_{t}^{2} d t<+\infty, \quad \int_{0}^{+\infty} e^{-\mu t} v_{t}^{2} d t<+\infty
$$

Thus it follows that $\|\mathbb{L} y\|_{L^{2}} \leq M\|y\|_{H}$, which means that $\mathbb{L}$ is a bounded linear operator.

(2) In order to evaluate invertibility, we need to get that if $(\mathbb{L} y)(t)=0$, then $y(t)=0$. In fact, according to $(\mathbb{L} y)(t)=y^{\prime \prime}(t)-p y^{\prime}(t)-q y(t), y(t)$ can be expressed as $y(t)=c_{1} e^{\lambda_{1} t}+c_{2} e^{\lambda_{2} t}$, here $\lambda_{1}=\frac{p+\sqrt{p^{2}+4 q}}{2}>0, \lambda_{2}=\frac{p-\sqrt{p^{2}+4 q}}{2}<$ 0 . Noting that $y(t) \in H_{0}$, we get

$$
\lim _{\mathrm{t} \rightarrow+\infty} \frac{c_{1} e^{\lambda_{1} t}+c_{2} e^{\lambda_{2} t}}{e^{r t}}=0, \quad r \in\left[0, \lambda_{1}\right]
$$

which indicates $c_{1}=0$. On the other hand, in view of the fact that $\alpha y(0)-$ $\beta y^{\prime}(0)-\sum_{\mathrm{i}=1}^{n} k_{i} y\left(\xi_{i}\right)=0$, it follows that

$$
c_{2} \alpha-c_{2} \beta \lambda_{2}-c_{2} \sum_{i=1}^{n} k_{i} e^{\lambda_{2} \xi_{i}}=0,
$$

thus from (H2) we have $c_{2}=0$ and hence $y(t)=0$.

Therefore Eq. (1.1) is turned into the following operator equation in $H_{0}$

$$
(\mathbb{L} y)(t)=g(t, y(t)) .
$$

Let $K(t, s)$ be the reproducing kernel function of $H_{0}$. Then for $t_{i} \in[0,+\infty)$, we define $\psi_{i}(t) \in H_{0}$ by

$$
\psi_{i}(t) \triangleq(\mathbb{L} K(t,(\cdot)))\left(t_{i}\right)
$$

Lemma 1. If $S=\left\{t_{1}, t_{2}, \ldots\right\}$ be a dense subset on $[0,+\infty)$, then $\left\{\psi_{i}(t)\right\}_{i=1}^{\infty}$ is a complete system of $H_{0}$.

Proof. For every $i$, according to the reproducing property of $K(t, s)$,

$$
\begin{aligned}
0 & =\left\langle y(t), \psi_{i}(t)\right\rangle_{H}=\left\langle y(t),\left(\mathbb{L} K(t,(\cdot))\left(t_{i}\right)\right\rangle_{H}\right. \\
& =\mathbb{L}\left\langle y(t), K(t,(\cdot))\left(t_{i}\right)\right\rangle_{H}=\mathbb{L}(y(\cdot))\left(t_{i}\right)=(\mathbb{L} y)\left(t_{i}\right) .
\end{aligned}
$$

Noting that $t_{i} \in S$ and $S$ is a dense set, then $(\mathbb{L} y)(t)=0 . y(t) \equiv 0$ is hold from the existence of $\mathbb{L}^{-1}$. Namely, $\left\{\psi_{i}(t)\right\}_{i=1}^{\infty}$ is a complete system.

By the Gram-Schmidt process a normal orthogonal basis $\left\{\tilde{\psi}_{i}(t)\right\}_{i=1}^{\infty}$ of $H_{0}$ is given by

$$
\tilde{\psi}_{i}(t)=\sum_{k=1}^{i} \beta_{i k} \psi_{k}(t)
$$


Furthermore, we provide the complexity estimation of the orthogonal basis in Appendix.

Here the approximate solution of (3.1) is constructed by a function sequence:

$$
y_{n}(t)=\sum_{i=1}^{n} \sum_{k=1}^{i} \beta_{i k} g\left(t_{k}, \mathbb{P}_{k-1} y\left(t_{k}\right)\right) \tilde{\psi}_{i}(t),
$$

where $y(t)$ is the exact solution, $\mathbb{P}_{n}$ is an orthogonal projector from $H_{0}$ to $\operatorname{span}\left\{\tilde{\psi}_{1}, \tilde{\psi}_{2}, \ldots, \tilde{\psi}_{n}\right\}$ and $\mathbb{P}_{0} y$ denotes any fixed function in $H_{0}$.

Lemma 2. Suppose $\left\{t_{i}\right\}_{i=1}^{\infty}$ is dense on $[0,+\infty)$. Then $y_{n}(t)$ in (3.3) is convergent to the $y(t)$.

Proof. Suppose $j \leq n$, due to

$$
\begin{aligned}
\mathbb{L} y_{n}\left(t_{j}\right) & =\sum_{i=1}^{n} \sum_{k=1}^{i} \beta_{i k} g\left(t_{k}, \mathbb{P}_{k-1} y\left(t_{k}\right)\right) \mathbb{L} \tilde{\psi}_{i}\left(t_{j}\right) \\
& =\sum_{i=1}^{n} \sum_{k=1}^{i} \beta_{i k} g\left(t_{k}, \mathbb{P}_{k-1} y\left(t_{k}\right)\right)\left\langle\tilde{\psi}_{i}, \mathbb{L} K\left(t, t_{j}\right)\right\rangle_{H} \\
& =\sum_{i=1}^{n} \sum_{k=1}^{i} \beta_{i k} g\left(t_{k}, \mathbb{P}_{k-1} y\left(t_{k}\right)\right)\left\langle\tilde{\psi}_{i}, \psi_{j}\right\rangle_{H}
\end{aligned}
$$

it follows that

$$
\begin{aligned}
\sum_{p=1}^{j} \beta_{j p} \mathbb{L} y_{n}\left(t_{p}\right) & =\sum_{i=1}^{n} \sum_{k=1}^{i} \beta_{i k} g\left(t_{k}, \mathbb{P}_{k-1} y\left(t_{k}\right)\right)\left\langle\tilde{\psi}_{i}, \sum_{p=1}^{j} \beta_{j p} \psi_{p}\right\rangle_{H} \\
& =\sum_{i=1}^{n} \sum_{k=1}^{i} \beta_{i k} g\left(t_{k}, \mathbb{P}_{k-1} y\left(t_{k}\right)\right)\left\langle\tilde{\psi}_{i}, \tilde{\psi}_{j}\right\rangle_{H} \\
& =\sum_{k=1}^{j} \beta_{j k} g\left(t_{k}, \mathbb{P}_{k-1} y\left(t_{k}\right)\right) .
\end{aligned}
$$

Considering $j=1$, we have $\left(\mathbb{L} y_{n}\right)\left(t_{1}\right)=g\left(t_{1}, \mathbb{P}_{0} y\left(t_{1}\right)\right)$. When $j=2$, then

$$
\beta_{21}\left(\mathbb{L} y_{n}\right)\left(t_{1}\right)+\beta_{22}\left(\mathbb{L} y_{n}\right)\left(t_{2}\right)=\beta_{21} g\left(t_{1}, \mathbb{P}_{0} y\left(t_{1}\right)\right)+\beta_{22} g\left(t_{2}, \mathbb{P}_{1} y\left(t_{2}\right)\right) .
$$

One gets that $\left(\mathbb{L} y_{n}\right)\left(t_{2}\right)=g\left(t_{2}, \mathbb{P}_{1} y\left(t_{2}\right)\right)$. Hence it can be obtained by induction

$$
\left(\mathbb{L} y_{n}\right)\left(t_{j}\right)=g\left(t_{j}, \mathbb{P}_{j-1} y\left(t_{j}\right)\right) .
$$

Since $\left\{t_{i}\right\}_{i=1}^{\infty}$ is dense, for any $t \in[0,+\infty)$ there exists a subsequence $\left\{t_{n_{j}}\right\}_{j=1}^{\infty}$ such that $t_{n_{j}} \rightarrow t$, as $j \rightarrow \infty$. Based on the convergence of $\mathbb{P}_{n}$ and the continuity of $g(\cdot, \cdot)$, it follows that

$$
\begin{aligned}
\lim _{j \rightarrow+\infty}\left(\mathbb{L} y_{n}\right)\left(t_{n_{j}}\right) & =\lim _{j \rightarrow+\infty} g\left(t_{n_{j}}, \mathbb{P}_{n_{j}-1} y\left(t_{n_{j}}\right)\right) \\
& =g(t, y(t))=\mathbb{L} y(t) .
\end{aligned}
$$


Moreover, according to (3.3) we have

$$
\begin{aligned}
\lim _{j \rightarrow+\infty}\left(\mathbb{L} y_{n}\right)\left(t_{n_{j}}\right) & =\lim _{j \rightarrow+\infty} \sum_{i=1}^{n} \sum_{k=1}^{i} \beta_{i k} g\left(t_{k}, \mathbb{P}_{k-1} y\left(t_{k}\right)\right) \mathbb{L} \tilde{\psi}_{i}\left(t_{n_{j}}\right) \\
& =\sum_{i=1}^{\infty} \sum_{k=1}^{i} \beta_{i k} g\left(t_{k}, \mathbb{P}_{k-1} y\left(t_{k}\right)\right) \mathbb{L} \tilde{\psi}_{i}(t) \\
& =\lim _{n \rightarrow+\infty} \mathbb{L} \sum_{i=1}^{n} \sum_{k=1}^{i} \beta_{i k} g\left(t_{k}, \mathbb{P}_{k-1} y\left(t_{k}\right)\right) \tilde{\psi}_{i}(t)=\lim _{n \rightarrow+\infty} \mathbb{L} y_{n}(t) .
\end{aligned}
$$

Therefore, we get

$$
\lim _{n \rightarrow+\infty} \mathbb{L} y_{n}(t)=\mathbb{L} y(t)
$$

We can use (3.4) to prove that $\mathbb{L} y_{n}(t)$ converges to $\mathbb{L} y(t)$ in $L^{2}$. This together with the boundedness of $\mathbb{L}^{-1}$ implies that

$$
\lim _{n \rightarrow+\infty} y_{n}(t)=\mathbb{L}^{-1} \lim _{n \rightarrow+\infty}\left(\mathbb{L} y_{n}(t)\right)=\mathbb{L}^{-1}(\mathbb{L} y(t))=y(t) .
$$

This completes the proof of the convergence of formula (3.3).

Thus, we claim that

$$
y(t)=\lim _{n \rightarrow+\infty} y_{n}(t)=\sum_{i=1}^{\infty} \sum_{k=1}^{i} \beta_{i k} g\left(t_{k}, \mathbb{P}_{k-1} y\left(t_{k}\right)\right) \tilde{\psi}_{i}(t) .
$$

Furthermore, from the property of orthogonal projector $\mathbb{P}_{n}$, it follows from (3.5) that

$$
\mathbb{P}_{n} y(t)=\sum_{i=1}^{n} \sum_{k=1}^{i} \beta_{i k} g\left(t_{k}, \mathbb{P}_{k-1} y\left(t_{k}\right)\right) \tilde{\psi}_{i}(t)=y_{n}(t),
$$

and consequently, $y_{n}(t)$ is the orthogonal projection of $y(t)$. Substituting $\mathbb{P}_{n} y(t)=y_{n}(t)$ into $(3.3)$, we get

$$
y_{n}(t)=\sum_{i=1}^{n} \sum_{k=1}^{i} \beta_{i k} g\left(t_{k}, y_{k-1}\left(t_{k}\right)\right) \tilde{\psi}_{i}(t)
$$

where $y_{0}(t)=\mathbb{P}_{0} y(t) \in H_{0}$.

Theorem 4. The approximate solution $y_{n}(t)$ and its derivatives $y_{n}^{\prime}(t), y_{n}^{\prime \prime}(t)$ inner-closed uniformly converge to exact solution $y(t)$ and its derivatives $y^{\prime}(t)$, $y^{\prime \prime}(t)$ on $[0,+\infty)$, respectively.

Proof. For any $t \in[0, T] \subset[0,+\infty), i=0,1,2$, using the boundedness of $\left\|\partial_{t}^{i} K(t, s)\right\|_{H}$ and reproducing property of $K(t, s)$, we have

$$
\begin{aligned}
\left|y_{n}^{(i)}(t)-y^{(i)}(t)\right| & =\left|\left(y_{n}(t)-y(t)\right)^{(i)}\right|=\left|\partial_{t}^{i}\left\langle y_{n}(s)-y(s), K(t, s)\right\rangle_{H}\right| \\
& =\left|\left\langle y_{n}(s)-y(s), \partial_{t}^{i} K(t, s)\right\rangle_{H}\right| \\
& \leq\left\|y_{n}-y\right\|_{H}\left\|\partial_{t}^{i} K(t, s)\right\|_{H} \leq M\left\|y_{n}-y\right\|_{H} \rightarrow 0 .
\end{aligned}
$$


Theorem 5. $\left|y_{n}(t)-y(t)\right|=o(1 / n)$.

Proof. Firstly, by using the denseness, for any $t \in[0,+\infty)$ and $n \in N$, we can take $t_{i} \in\left\{t_{1}, t_{2}, \ldots\right\}, i \leq n$ such that $\left|t-t_{i}\right|<1 / n$. Using the reproducing property, we obtain

$$
y_{n}(t)=\left\langle y_{n}(\cdot), K(t, \cdot)\right\rangle_{H} \cdot
$$

With the help of $(3.7)$, we now get $\mathbb{L} y_{n}(t)=\left\langle y_{n}(\cdot), \mathbb{L} K(t, \cdot)\right\rangle_{H}$. Together with the property of projector yields

$$
\begin{aligned}
\mathbb{L} y_{n}\left(t_{i}\right) & =\left\langle y_{n}(\cdot), \mathbb{L} K\left(t_{i}, \cdot\right)\right\rangle_{H}=\left\langle\mathbb{P}_{n} y(\cdot), \psi_{i}(\cdot)\right\rangle_{H}=\left\langle y(\cdot), \mathbb{P}_{n} \psi_{i}(\cdot)\right\rangle_{H} \\
& =\left\langle y(\cdot), \psi_{i}(\cdot)\right\rangle_{H}=\mathbb{L}\left\langle y(\cdot), K\left(t_{i}, \cdot\right)\right\rangle_{H}=\mathbb{L} y\left(t_{i}\right) .
\end{aligned}
$$

Therefore

$$
\begin{aligned}
\mathbb{L} y_{n}(t)-\mathbb{L} y(t) & =\mathbb{L} y_{n}(t)-\mathbb{L} y_{n}\left(t_{i}\right)-\left(\mathbb{L} y(t)-\mathbb{L} y\left(t_{i}\right)\right) \\
& =\left\langle y_{n}(\cdot), \mathbb{L} K(t, \cdot)-\mathbb{L} K\left(t_{i}, \cdot\right)\right\rangle_{H}-\left\langle y(\cdot), \mathbb{L} K(t, \cdot)-\mathbb{L} K\left(t_{i}, \cdot\right)\right\rangle_{H} \\
& =\left\langle y_{n}(\cdot)-y(\cdot), \mathbb{L} K(t, \cdot)-\mathbb{L} K\left(t_{i}, \cdot\right)\right\rangle_{H} .
\end{aligned}
$$

By the mean value theorem, we have

$$
\mathbb{L} K(t, \cdot)-\mathbb{L} K\left(t_{i}, \cdot\right)=\left(t-t_{i}\right) \frac{\partial}{\partial s} \mathbb{L} K(s, \cdot) .
$$

Finally, the following conclusion follows from above:

$$
\begin{aligned}
\left|y_{n}(t)-y(t)\right| & =\left\langle y_{n}-y, \mathbb{L}^{-1}\left(\mathbb{L} K(t, \cdot)-\mathbb{L} K\left(t_{i}, \cdot\right)\right)\right\rangle_{H} \\
& \leq\left\|\mathbb{L}^{-1}\right\|_{H}\left\|y_{n}-y\right\|_{H}\left\|\mathbb{L} K(t, \cdot)-\mathbb{L} K\left(t_{i}, \cdot\right)\right\|_{H} \\
& \leq\left\|\mathbb{L}^{-1}\right\|_{H}\left\|y_{n}-y\right\|_{H}\left|t-t_{i}\right|\left\|\frac{\partial}{\partial s} \mathbb{L} K(s, \cdot)\right\|_{H} .
\end{aligned}
$$

Thus, according to $\left\|y_{n}-y\right\|_{H} \rightarrow 0,\left|t-t_{i}\right|<1 / n$ and the boundedness of $\left\|\frac{\partial}{\partial s} \mathbb{L} K(s, \cdot)\right\|_{H}$, we get $\left|y_{n}(t)-y(t)\right|=o(1 / n)$.

\section{Numerical Example}

In this section we will give some numerical examples of multi-point boundary value problem that show the exactness and usefulness of our presented process.

Example 1. Consider a system of second-order nonlinear multi-point boundary value problem as following:

$$
\left\{\begin{array}{l}
y^{\prime \prime}(t)-y^{\prime}(t)-2 y(t)+e^{-3 t}\left(\sin y(t)+169 t^{4}+62 t^{3}+18 t^{2}+19\right)=0 \\
10 y(0)-\frac{1}{4} y^{\prime}(0)-y\left(\frac{1}{2}\right)-\frac{1}{9} y(2)=0, \quad \lim _{t \rightarrow+\infty} \frac{y(t)}{e^{t}}=0
\end{array}\right.
$$

Applying the method presented in Section 3, we calculate the approximate solution $y_{n}(t)$ of Eq. (4.1) as following: 
Table 1. $\left|\mathbb{L} x_{n}(t)-f\left(t, x_{n}(t)\right)\right|$ for Example 1.

\begin{tabular}{rlll}
\hline \multicolumn{1}{c}{$t$} & $k=1, h=5 / 4$ & $k=2, h=5 / 8$ & $k=4, h=5 / 32$ \\
\hline 5.00 & $2.53 \mathrm{E}-10$ & $1.27 \mathrm{E}-12$ & $4.19 \mathrm{E}-13$ \\
2.50 & $1.85 \mathrm{E}-09$ & $1.07 \mathrm{E}-09$ & $2.56 \mathrm{E}-13$ \\
3.75 & $1.48 \mathrm{E}-07$ & $1.05 \mathrm{E}-11$ & $3.70 \mathrm{E}-12$ \\
6.25 & $4.33 \mathrm{E}-12$ & $5.82 \mathrm{E}-13$ & $8.61 \mathrm{E}-12$ \\
7.50 & $3.19 \mathrm{E}-12$ & $1.69 \mathrm{E}-12$ & $2.37 \mathrm{E}-12$ \\
8.75 & $5.85 \mathrm{E}-13$ & $1.35 \mathrm{E}-11$ & $2.37 \mathrm{E}-12$ \\
10.00 & $2.29 \mathrm{E}-12$ & $1.62 \mathrm{E}-12$ & $3.02 \mathrm{E}-12$ \\
3.13 & & $1.24 \mathrm{E}-10$ & $1.32 \mathrm{E}-12$ \\
4.38 & & $1.88 \mathrm{E}-12$ & $6.32 \mathrm{E}-13$ \\
5.63 & & $8.20 \mathrm{E}-12$ & $9.72 \mathrm{E}-13$ \\
6.88 & & $1.53 \mathrm{E}-12$ & $1.09 \mathrm{E}-12$ \\
10.63 & & $5.60 \mathrm{E}-13$ & $4.07 \mathrm{E}-13$ \\
11.25 & & $7.18 \mathrm{E}-12$ & $4.61 \mathrm{E}-12$ \\
12.50 & & $1.19 \mathrm{E}-11$ & $6.98 \mathrm{E}-12$ \\
13.13 & & $5.22 \mathrm{E}-12$ & $6.66 \mathrm{E}-12$ \\
14.38 & & $1.67 \mathrm{E}-11$ & $7.35 \mathrm{E}-12$ \\
15.00 & & $9.60 \mathrm{E}-12$ & $2.74 \mathrm{E}-13$ \\
2.81 & & & $7.65 \mathrm{E}-13$ \\
8.44 & & & $2.73 \mathrm{E}-12$ \\
11.72 & & & $7.58 \mathrm{E}-12$ \\
16.06 & & & $4.44 \mathrm{E}-11$ \\
20.16 & & & $9.26 \mathrm{E}-11$ \\
22.50 & & & $1.41 \mathrm{E}-11$ \\
23.13 & & & $1.09 \mathrm{E}-11$ \\
25.00 & & & $1.81 \mathrm{E}-12$ \\
\hline $\mathrm{CPU}$ & & & 83.922 \\
\hline & & &
\end{tabular}

1. A weighted reproducing kernel Hilbert space is constructed to solve the problem (4.1) and the reproducing kernel can be calculated by formula (2.1).

2. Choosing a dense subset $S=\left\{t_{i}\right\}_{i=1}^{\infty}$ in $[0,+\infty), t_{1}=a>0(a=5)$, $t_{2}=\frac{a}{2}, t_{k 2^{k}+m}=\frac{2 m-1}{2^{k+1}} a\left(1 \leq m \leq k 2^{k}\right), t_{k 2^{k+1}+n}=\frac{k 2^{k+1}+n}{2^{k+1}} a\left(1 \leq n \leq 2^{k+1}\right)$, $k=1,2,3 \ldots$, it can be seen that $S_{k}=\left\{t_{1}, t_{2}, \ldots, t_{(k+1) 2^{k+1}}\right\} \subset S$,

$$
\lim _{k \rightarrow+\infty} t_{(k+1) 2^{k+1}}=\lim _{k \rightarrow+\infty}(k+1) a=+\infty
$$

and for the step of $S_{k}$,

$$
\lim _{k \rightarrow+\infty} h_{k}=\lim _{k \rightarrow+\infty} a / 2^{k+1}=0 .
$$

By using (3.2) we get the orthogonalization coefficients $\beta_{i k}$ and $\tilde{\psi}_{i}(t)$.

3. Then taking $y_{0}(t)=0$ the approximate solution $y_{n}(t)$ can be given iteratively by formula (3.6). The absolute errors for $k=1,2,4$ illustrate that accuracy of numerical computation is higher (see Table 1). Also it explains clear superiority of CPU time from Table 1.

Example 2. Consider the following special case of Eq. (1.1) with three-point 
Table 2. $\left|\mathbb{L} x_{n}(t)-f\left(t, x_{n}(t)\right)\right|$ for Example 2 .

\begin{tabular}{|c|c|c|c|}
\hline$t$ & $k=1, h=3 / 2$ & $k=4, h=3 / 16$ & $k=5, h=3 / 32$ \\
\hline 6.00 & 3.20 E-13 & 4.55 E-14 & $1.39 \mathrm{E}-14$ \\
\hline 3.00 & 3.55 E-13 & 3.27 E-13 & $3.41 \mathrm{E}-13$ \\
\hline 1.50 & 8.88 E-13 & 9.95 E-14 & 6.75 E-14 \\
\hline 4.50 & $1.03 \mathrm{E}-12$ & $1.69 \mathrm{E}-13$ & $1.27 \mathrm{E}-13$ \\
\hline 7.50 & 2.17 E-12 & $1.31 \mathrm{E}-12$ & $1.24 \mathrm{E}-14$ \\
\hline 9.00 & $5.50 \mathrm{E}-12$ & 3.84 E-12 & 3.76 E-12 \\
\hline 10.25 & $1.99 \mathrm{E}-12$ & 5.83 E-12 & $2.37 \mathrm{E}-12$ \\
\hline 12.00 & $4.12 \mathrm{E}-12$ & 3.94 E-13 & 3.13 E-13 \\
\hline 0.75 & & 5.77 E-14 & $1.29 \mathrm{E}-14$ \\
\hline 2.25 & & 1.74 E-13 & $5.33 \mathrm{E}-13$ \\
\hline 8.25 & & 3.48 E-12 & 3.57 E-12 \\
\hline 15.00 & & 4.94 E-11 & $4.60 \mathrm{E}-11$ \\
\hline 18.00 & & 8.42 E-11 & $9.33 \mathrm{E}-11$ \\
\hline 21.00 & & 7.49 E-11 & 2.47 E-11 \\
\hline 23.63 & & $1.40 \mathrm{E}-11$ & 7.87 E-12 \\
\hline 0.19 & & $6.26 \mathrm{E}-13$ & $1.32 \mathrm{E}-13$ \\
\hline 5.44 & & $1.01 \mathrm{E}-12$ & $1.28 \mathrm{E}-13$ \\
\hline 30.00 & & 4.93 E-11 & $1.69 \mathrm{E}-11$ \\
\hline 0.09 & & & $1.31 \mathrm{E}-13$ \\
\hline 1.03 & & & 1.77 E-15 \\
\hline 7.22 & & & $1.67 \mathrm{E}-12$ \\
\hline 29.16 & & & $2.92 \mathrm{E}-11$ \\
\hline 33.56 & & & $1.38 \mathrm{E}-11$ \\
\hline 35.81 & & & $3.41 \mathrm{E}-11$ \\
\hline 36.00 & & & $1.09 \mathrm{E}-11$ \\
\hline CPU & 0.34 & 11.218 & 69.734 \\
\hline
\end{tabular}

boundary condition:

$$
\left\{\begin{array}{l}
y^{\prime \prime}(t)-2 y^{\prime}(t)-3 y(t)+9 t+t^{2}-\sqrt{y(t)}=0, \quad t>0 \\
y(0)-2 y(1)=0, \quad \lim _{t \rightarrow+\infty} \frac{y^{\prime}(t)}{e^{t}}=0
\end{array}\right.
$$

According to the numerical algorithm given above, the numerical solution of this problem is obtained by the improvement of RKM on the half-line. From Table 2, our approach clearly yields a reliable numerical solution. We also list the CPU cost in Table 2.

\section{Appendix}

In this section, the estimates of the complexity for construction the orthogonal basis are discussed. We know that the orthogonal basis $\tilde{\psi}_{i}(t)=\sum_{k=1}^{i} \beta_{i k} \psi_{k}(t)$ is obtained by orthogonalization of complete system. The algorithm with time complexity may be analyzed as following:

Step 1: Computing $\left\langle\psi_{i}(t), \psi_{k}(t)\right\rangle_{H}$. In fact, according to the properties of reproducing kernel and bounded linear operator, we have

$$
\left\langle\psi_{i}(t), \psi_{k}(t)\right\rangle_{H}=\mathbb{L} \psi_{i}\left(t_{k}\right) .
$$


Thus, we only need to calculate the specific function value $\mathbb{L} \psi_{i}\left(t_{k}\right)$ to avoid the complex integral. In order to calculate the approximate solution $y_{n}(t)$ for Eq. (3.1), it takes $\frac{n(n+1)}{2}$ times of computing time of the specific function value.

Step 2: Orthogonalization can be obtained by the following cycle:

(1) Let $\beta_{11}=\frac{1}{\left\|\psi_{1}\right\|}$

(2) Consider $2 \leq \mathrm{i} \leq \mathrm{n}$. For $1 \leq j \leq i-1$, we denote

$$
C_{i j}=\left\langle\psi_{i}, \tilde{\psi}_{j}\right\rangle_{H}=\left\langle\psi_{i}, \sum_{k=1}^{i} \beta_{j k} \psi_{k}\right\rangle_{H}=\sum_{k=1}^{i} \beta_{j k}\left\langle\psi_{i}, \psi_{k}\right\rangle_{H}
$$

It needs $\frac{i(i-1)}{2}$ multiplication operations to compute $C_{i j}$.

For $1 \leq m \leq i-1$, we denote $\beta_{i m}=-\sum_{k=m}^{i-1} C_{i k} \beta_{k m} /\left\|\tilde{\psi}_{i}\right\|$, where

$$
\left\|\tilde{\psi}_{i}\right\|=\left(\left\|\psi_{i}\right\|^{2}-\sum_{k=1}^{i-1}\left|C_{i k}\right|^{2}\right)^{1 / 2}=\left(\left\langle\psi_{i}, \psi_{i}\right\rangle_{H}-\sum_{k=1}^{i-1} C_{i k}^{2}\right)^{1 / 2}
$$

Here $\frac{(i+2)(i-1)}{2}$ multiplication and division operations are required to compute $\beta_{i m}$.

The cycle in (2) uses $\frac{n(n-1)(2 n+3)}{4}$ multiplication and division operations.

To sum up, from Step 1 and Step 2 we get that the total orthogonal basis complexity is given by $\frac{n(n-1)(2 n+3)}{4}$ multiplication and division operations plus $\frac{n(n+1)}{2}$ operations for computing the specific function value. Therefore, the construction of orthogonal basis costs a total of $O\left(n^{3}\right)$ operations.

\section{References}

[1] R.P. Agarwal and D. O'Regan. Infinite Interval Problems for Differential, Difference and Integral Equations. Kluwer Academic, 2001.

[2] S. Cano-Casanova and J. Lopez-Gomez. Existence, uniqueness and blow-up rate of large solutions for a canonical class of one-dimensional problems on the halfline. J. Differential Equations, 244:3180-3203, 2008. http://dx.doi.org/10.1016/j.jde.2007.11.012.

[3] Z. Chen and Y.Z. Lin. The exact solution of a linear integral equation with weakly singular kernel. J. Math. Anal. Appl., 344:726-734, 2008. http://dx.doi.org/10.1016/j.jmaa.2008.03.023.

[4] M.G. Cui and Y.Z. Lin. Nonlinear Numerical Analysis in Reproducing Kernel Hilbert Space. Nova Science Publisher, New York, 2009.

[5] S. Djebali and K. Mebarki. Multiple unbounded positive solutions for three-point BVPs with sign-changing nonlinearities on the positive half-line. Acta. Appl. Math., 109:361-388, 2010. http://dx.doi.org/10.1007/s10440-008-9322-3.

[6] H. Lian and W.G. Ge. Existence of positive solutions for Sturm-Liouville boundary value problems on the half-line. J. Math. Anal. Appl., 321:781-792, 2006. http://dx.doi.org/10.1016/j.jmaa.2005.09.001. 
[7] H. Lian, H.H. Pang and W.G. Ge. Triple positive solutions for boundary value problems on infinite intervals. Nonlinear Anal., 67:2199-2207, 2007. http://dx.doi.org/10.1016/j.na.2006.09.016.

[8] Y.Z. Lin and Y.F. Zhou. Solving nonlinear pseudoparabolic equations with nonlocal boundary conditions in reproducing kernel space. Numer. Algorithms, 52:173-186, 2009. http://dx.doi.org/10.1007/s11075-009-9263-6.

[9] B.M. Liu, L.S. Liu and Y.H. Wu. Unbounded solutions for three-point boundary value problems with nonlinear boundary conditions on $[0,+\infty)$. Nonlinear Anal., 73:2923-2932, 2010. http://dx.doi.org/10.1016/j.na.2010.06.052.

[10] J.D. Murray. Mathematical Biology. Springer, Berlin, 1989.

[11] D. O'Regan. Theory of Singular Boundary Value Problems. World Scientific, Singapore, 1994.

[12] D. O'Regan, B. Yan and R.P. Agarwal. Solutions in weighted spaces of singular boundary value problem on the half-line. J. Comput. Appl. Math., 205:751-763, 2007. http://dx.doi.org/10.1016/j.cam.2006.02.055.

[13] Y. Tian and W.G. Ge. Positive solutions for multi-point boundary value problem on the half-line. J. Math. Anal. Appl., 325:1339-1349, 2007. http://dx.doi.org/10.1016/j.jmaa.2006.02.075.

[14] B. Yan, D. O'Regan and R.P. Agarwal. Unbounded solutions for singular boundary value problems on the semi-infinite interval: Upper and lower solutions and multiplicity. J. Comput. Appl. Math., 197:365-386, 2006. http://dx.doi.org/10.1016/j.cam.2005.11.010.

[15] Y.F. Zhou, M.G. Cui and Y.Z. Lin. A computational method for nonlinear 2m-th order boundary value problems. Math. Model. Anal., 15:571-586, 2010. http://dx.doi.org/10.3846/1392-6292.2010.15.571-586. 